\title{
Identification of transcription factor co-regulators that drive prostate cancer progression
}

\author{
Manjunath Siddappa ${ }^{1}$, Sajad A. Wani ${ }^{1}$, Mark D. Long ${ }^{2}$, Damien A. Leach ${ }^{3}$, Ewy A. Mathé ${ }^{4,5}$, \\ Charlotte L. Bevan ${ }^{3}$ \& Moray J. Campbell1,6,7凶
}

In prostate cancer (PCa), and many other hormone-dependent cancers, there is clear evidence for distorted transcriptional control as disease driver mechanisms. Defining which transcription factor (TF) and coregulators are altered and combine to become oncogenic drivers remains a challenge, in part because of the multitude of TFs and coregulators and the diverse genomic space on which they function. The current study was undertaken to identify which TFs and coregulators are commonly altered in PCa. We generated unique lists of TFs $(n=2662)$, coactivators (COA; $n=766$ ); corepressors (COR; $n$ = 599); mixed function coregulators (MIXED; $n=511$ ), and to address the challenge of defining how these genes are altered we tested how expression, copy number alterations and mutation status varied across seven prostate cancer (PCa) cohorts (three of localized and four advanced disease). Testing of significant changes was undertaken by bootstrapping approaches and the most significant changes were identified. For one commonly and significantly altered gene were stably knockeddown expression and undertook cell biology experiments and RNA-Seq to identify differentially altered gene networks and their association with PCa progression risks. COAS, CORS, MIXED and TFs all displayed significant down-regulated expression (q.value $<0.1$ ) and correlated with protein expression (r 0.4-0.55). In localized PCa, stringent expression filtering identified commonly altered TFs and coregulator genes, including well-established (e.g. ERG) and underexplored (e.g. PPARGC1A, encodes PGC1 $\alpha$ ). Reduced PPARGC1A expression significantly associated with worse disease-free survival in two cohorts of localized PCa. Stable PGC1 $\alpha$ knockdown in LNCaP cells increased growth rates and invasiveness and RNA-Seq revealed a profound basal impact on gene expression ( 2300 genes; FDR < 0.05, logFC > 1.5), but only modestly impacted PPARy responses. GSEA analyses of the PGC1 $\alpha$ transcriptome revealed that it significantly altered the AR-dependent transcriptome, and was enriched for epigenetic modifiers. PGC1 $\alpha$-dependent genes were overlapped with PGC1 $\alpha$-ChIP-Seq genes and significantly associated in TCGA with higher grade tumors and worse disease-free survival. These methods and data demonstrate an approach to identify cancer-driver coregulators in cancer, and that PGC1 $\alpha$ expression is clinically significant yet underexplored coregulator in aggressive early stage $\mathrm{PCa}$.

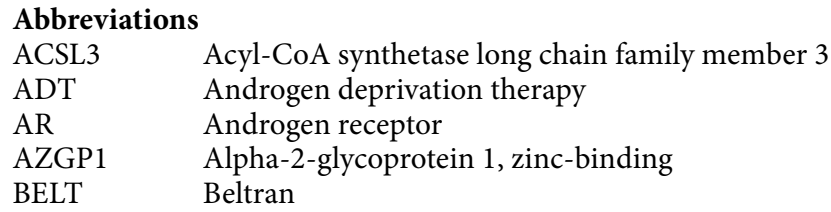

${ }^{1}$ College of Pharmacy, Pharmaceutics and Pharmaceutical Chemistry, The Ohio State University, 536 Parks Hall, 500 West 12th Ave, Columbus, OH 43210, USA. ${ }^{2}$ Department of Cancer Genetics and Genomics, Roswell Park Comprehensive Cancer Center (RPCCC), Buffalo, NY 14263, USA. ${ }^{3}$ Department of Surgery and Cancer, Imperial Centre for Translational and Experimental Medicine, Imperial College London, Hammersmith Hospital, Du Cane Road, London W12 0NN, UK. " Biomedical Informatics Department, College of Medicine, The Ohio State University, Columbus, $\mathrm{OH}$ 43210, USA. ${ }^{5}$ Division of Preclinical Innovation, National Center for Advancing Translational Sciences, NIH, 9800 Medical Center Dr, Rockville, MD 20892, USA. ${ }^{6}$ The James, Comprehensive Cancer Center, The Ohio State University, Columbus, OH 43210, USA. ${ }^{7}$ Biomedical Informatics Shared Resource, The Ohio State University, Columbus, OH 43210, USA. ${ }^{\boxplus}$ email: Campbell.1933@OSU.edu 


$\begin{array}{ll}\text { CNA } & \text { Copy number alterations } \\ \text { COA } & \text { Coactivators } \\ \text { COR } & \text { Corepressors } \\ \text { DDX58 } & \text { DExD/H-Box Helicase 58 } \\ \text { DHRS3 } & \text { Retinoic acid anabolizing enzyme, dehydrogenase/reductase 3 } \\ \text { GSEA } & \text { Gene set enrichment analyses } \\ \text { ERG } & \text { ETS transcription factor } \\ \text { ETYA } & \text { 5,8,11,14-Eicosatetraynoic acid } \\ \text { FHCRC } & \text { Fred Hutchinson Cancer Research Center } \\ \text { GDF15 } & \text { Growth differentiation factor 15 } \\ \text { HERPUD1 } & \text { Homocysteine inducible ER protein with ubiquitin like domain 1 } \\ \text { HOX } & \text { Homeobox } \\ \text { HPGD } & \text { Hydroxyprostaglandin dehydrogenase } \\ \text { KDM } & \text { Lysine demethylases } \\ \text { KS test } & \text { Kolmogorov-Smirnov test } \\ \text { MICH } & \text { University of Michigan } \\ \text { MIXED } & \text { Mixed function coregulators } \\ \text { MSKCC } & \text { Memorial Sloan Kettering Cancer Center } \\ \text { NR3C1/GR } & \text { Glucocorticoid receptor } \\ \text { OICR } & \text { Ontario Institute for Cancer Research } \\ \text { PCa } & \text { Prostate cancer } \\ \text { PPAR } \gamma & \text { Peroxisome proliferator-activated receptor gamma } \\ \text { PPARGC1A } & \text { Peroxisome proliferator-activated receptor gamma coactivator 1-alpha } \\ \text { PGC1a } & \text { Peroxisome proliferator-activated receptor gamma coactivator 1-alpha (protein) } \\ \text { SU2C } & \text { Stand up two caner } \\ \text { TCGA } & \text { The cancer genome atlas } \\ \text { TF } & \text { Transcription factor } \\ \text { UGT2B17 } & \text { UDP glucuronosyltransferase family 2 member B17 } \\ & \end{array}$

Prostate cancer (PCa) is a high-profile hormone-responsive cancer that displays uncertain progression risks. For men with localized PCa, it is unclear which patients will experience treatment failure following either surgery or radiotherapy. In advanced $\mathrm{PCa}$, it's similarly unclear which patients will experience a sustained response to androgen deprivation therapy (ADT), and who will experience treatment failure giving rise to ADT-recurrent PCa (ADT-RPCa).

Many of these prognostic uncertainties arise due to changes in the control of androgen receptor (AR) signaling. In the normal prostate gland the AR functions in a large and dynamic multimeric complex to regulate gene expression programs that control tissue homeostasis and epithelial cell fates ${ }^{1,2}$. In PCa there are strong examples of how signaling is distorted due to changes in AR expression and structural variation, alongside changes in the members of the AR complex ${ }^{3-13}$. Furthermore, epigenomic events ${ }^{14-18}$ alter enhancer accessibility of downstream AR target genes and even upstream of the AR gene itself ${ }^{19}$. As a result of these changes to the AR complex and allied epigenetic events, the normal differentiation driving and growth limiting functions of the AR are attenuated and there is enhanced regulation of genes that promote aggressive cancer phenotype ${ }^{20-23}$.

This re-wiring of AR signaling is mirrored by disrupted functions of other members of the nuclear hormone receptor super-family ${ }^{11,24}$, and probably occurs more widely to alter how transcription factors (TFs) function. Re-wiring of TFs changes the consequences of cell signaling and contributes to changes in lineage plasticity that are also associated with aggressive $\mathrm{PCa}^{25-27}$. Therefore understanding how TF signaling occurs in PCa progression can lead to the development of strategies for targeting these changes through targeted epigenetic therapies ${ }^{28-30}$, or the downstream gene networks may be uniquely drug-sensitive ${ }^{31,32}$. Furthermore disease-specific enhancers that are newly activated provide rationale for targeted deep sequencing to dissect the interactions of germline and structural variation at tumor-driving enhancers ${ }^{33,34}$. These studies have revealed a number of high profile and common alterations in TFs and coregulators in PCa. For example, ERG ${ }^{35}$ and NR3C1/GR ${ }^{36}$ have been identified as commonly altered, whereas others such as the PPAR $\gamma$ coactivator PGC1 $\alpha^{37,38}$ are relatively underinvestigated. However, given the multitude of TFs and coregulators, many have not been investigated at all in the context of PCa.

The Cancer Genome Atlas (TCGA) and other consortia ${ }^{39-41}$ established mutation, copy number variation and gene/protein expression in hundreds of tumors from both early and advanced PCa along-side clinical features and patient outcome data. These studies have revealed powerful insights into PCa drivers, and offer up the possibility for secondary analyses focusing specifically on TFs and coregulators. In the current study TCGA and other genome-wide data were used specifically to test how TFs and their co-regulators are altered and associate with PCa outcome.

We utilized seven PCa cohorts; three cohorts of localized PCa (TCGA ${ }^{42}$, MSKCC $^{43}, \mathrm{OICR}^{41}$ ) and four cohorts of advanced PCa $\left(\mathrm{MICH}^{44} \mathrm{FHCRC}^{44}, \mathrm{BELT}^{45} \mathrm{SU} 2 \mathrm{C}^{46,47}\right)$. In these cohorts we examined the expression and genetic changes in different classes of coregulators and TFs, and within each class identified the most significant and clinically-relevant gene changes. These approaches established the class-specific significant mRNA and protein down-regulation of TFs and co-regulators in localized PCa, which were not further altered in advanced PCa, and are not altered significantly by either mutation or copy number alterations (CNA).

These approaches revealed that PPARGC1A (encodes PGC1 $\alpha$ ) was commonly down-regulated associated with worse disease outcome in local PCa. Stable knockdown of PGC1 $\alpha$ in LNCaP cells increased proliferation, led 
to a more invasive phenotype, and profoundly changed gene expression patterns both positively and negatively. Combining these data with differentially regulated genes between tumors with high or low PPARGC1A expression alongside PGC1a ChIP-Seq data identified a network of 60 genes that are both significantly bound and regulated by PGC1 $\alpha$ and significantly associated with higher Gleason Grade tumors. In parallel, these approaches establish a relatively generic work-flow for analyses of gene families or functional groupings to allow investigators to identify clinically-significant relationships which in turn support functional analyses.

\section{Materials and methods}

Data analyses, integration and code availability. PCa data was downloaded from $\mathrm{cBioPortal}^{40}$. Analyses was undertaken using $\mathrm{R}$ (version 3.6.2) ${ }^{48}$.

Cell culture and materials. LNCaP cells (ATCC) were confirmed to be mycoplasma free everyon month and authenticated using STR profiling. Cells were treated with 5,8,11,14-Eicosatetraynoic acid (ETYA).

Cell viability and scratch assays. Cellular viability was measured by bioluminescent detection of ATP (CellTitre-Glo assay kit (Promega)). Cells were plated at $5 \times 10^{3}$ cells per well in 96-well, white-walled plates, allowed to adhere and treated with ETYA $(10 \mu \mathrm{M})$ or EtOH (vehicle control) to a final volume of $100 \mu \mathrm{l}$ for $96 \mathrm{~h}$. Each experiment was performed in triplicate in triplicate wells. For scratch assay, cells were seeded at $1 \times 10^{6}$ cells per well in a 6-well plate, allowed to adhere for $48 \mathrm{~h}$ to a confluence of about $80 \%$ and then wounded by scratching with p200 sterile pipette tip. The debris were removed, and cells washed to make ensure the edges were smoothed with the same dimensions for experimental and control cells. Cells were incubated, and cell migration was assessed by monolayer gap closure after $48 \mathrm{~h}$.

Stable knockdown of PGC1 $\alpha$ in LNCaP Cells. PGC1a (encoded by PPARGC1A) was knockeddown with shRNA constructs (TG310260, Origene) in LNCaP cells (LNCaP_shPGC1A) or scrambled shRNA (LNCaP_shCtrl). Two PGC1A targeting constructs (shPGC1A-34 and shPGC1A-35) were selected and maintained in media containing puromycin $(0.2 \mathrm{ug} / \mathrm{ml})$.

Western immunoblotting. Total cellular protein was isolated from exponentially growing cells and lysed in ice cold RIPA buffer containing $1 \times$ cOmplete Mini Protease Inhibitor Tablets (Roche). Protein concentration were quantified and 75 ug resolved (SDS-PAGE) using $10 \%$ polyacrylamide gels, transferred to PVDF membrane and probed with primary antibody against PGC1a (PA5-72948, Invitrogen) and Beta-Actin (ab8229, abcam) overnight at $4{ }^{\circ} \mathrm{C}$. Primary antibody was detected with HRP-linked goat anti-rabbit IgG (abcam) and signal captured (ChemiDoc XRS + system (Bio-Rad)).

Unique lists of annotated transcription factors and co-regulators. A comprehensive list of TFs and co-regulators genes was developed by text-mining ${ }^{49}$ Gene Ontology (GO) terms that contained phrases including "positive control of transcription", "negative control of transcription", "co-activator", "co-repressor". From these GO terms, the HGNC gene name and ENSEMBL transcript ids were retrieved using biomaRt ${ }^{50}$ and combined with canonical lists of TFs from UniProt $(n=1994)$ and FANTOM ${ }^{67}(n=1988)$.

Groups were cross-referenced for uniqueness and annotated as following. TFs $(n=2662)$; coactivators were genes that exclusively associated with positive regulation of transcription (COA; $n=766$ ); corepressors were genes that exclusively associated with negative regulation of transcription (COR; $n=599$ ); mixed function coregulators were genes with evidence of context dependent negative and positive regulation of gene expression (MIXED; $\mathrm{n}=511)$ (Supplementary Table S1).

Testing family-wide changes in each gene category. Bootstrapping permutation approaches (boot), were used to test if the proportions of each gene group was altered more than predicted by chance. For mRNA and protein expression, Z-scores were calculated for gene expression data. In the cohorts of local tumor (MSKCC, TCGA) gene expression was calculated as Z-scores of tumor-normal comparisons with genes detectible in at least $80 \%$ of samples. In the other data sets the median expression data frame of RNA-Seq or Mass-Spec was converted to Z-scores. For copy number alterations (CNA) (via the GISTIC 2 method ${ }^{51}$ ) and mutations the data were used as obtained from cBioPortal ${ }^{40}$. To establish mutation frequency, the coding length of all exons for a given gene (including all alternative exons; BioMart) was calculated to yield the total gene CDS length. The mutation frequency was then calculated as the number of gene-length normalized mutations per gene and the square root of the summed mutation rate to control for the number of tumors in a cohort.

To test whether observed alteration frequencies within gene classes (e.g. COA, COR) was altered significantly, a vector of changes for all genes was calculated and the observed values (e.g. mean Z-score of expression) of a given class tested compared to all genes detected in each cancer cohort. Random sampling method was applied 100,000 times to select gene sets of equivalent size to simulate the distribution of changes across the genome for comparison ${ }^{52}$. Empirical p-values were calculated based on the group position relative to the sampling distribution of the genome.

Testing relationships between the most significantly altered transcription factor and co-regulators and clinical outcome. Gene expression levels in each gene family were filtered (genefilter) to select for genes that were commonly and significantly altered e.g. 2 Zscores in $\mathrm{x} \%$ of tumors (figure legends). The expression and clustering of genes were then visualized with pheatmap, and the intersections visualized with 


\begin{tabular}{|l|l|l|l|l|l|l|l|}
\hline Cohort & Tumor type & Material & Tumors & COAS & CORS & MIXED & TFs \\
\hline MSKCC & Local & RNA, CNA & 152 & 696 & 439 & 457 & 1506 \\
\hline TCGA & Local & RNA, CNA, exome muts & 498 & 663 & 415 & 414 & 1305 \\
\hline OICR & Local & Protein & 76 & 320 & 185 & 156 & 295 \\
\hline FH & Metastatic & RNA, CNA, exome muts & 148 & 712 & 445 & 465 & 1384 \\
\hline MICH & Metastatic & RNA, CNA, exome muts & 95 & 276 & 179 & 169 & 740 \\
\hline SU2C & Metastatic & RNA, CNA, exome muts & 271 & 543 & 343 & 384 & 1122 \\
\hline NEURO & Metastatic & RNA, CNA, exome muts & 51 & 725 & 466 & 485 & 1518 \\
\hline
\end{tabular}

Table 1. Summary of the prostate cancer cohorts. The number of tumors and the number for genes detected from each category in the unique gene lists (TFs $(n=2662)$; coactivator $(C O A ; n=766)$; corepressor $(C O R$; $\mathrm{n}=599$ ); mixed function coregulator (MIXED; $\mathrm{n}=511$ ).

$\mathrm{UpSetR}^{53}$ The association of patient cluster membership and clinical outcome (either categorical data or continuous data that was categorized) was then tested using a Chi-squared test and Kaplan-Meier curves generated for individual genes (survival) ${ }^{54}$.

Analyses of the PGC1 $\alpha$-dependent transcriptome and cistrome in cells and TCGA cohorts. LNCaP_shPGC1A and LNCaP_shCtrl cells $\left(1 \times 10^{6}\right.$ cells per well in a 6 -well plate $)$ adhered for $24 \mathrm{~h}$ then dosed with ETYA $(10 \mu \mathrm{M})$ or EtOH (vehicle control) and RNA extracted. All the samples were prepared in triplicates for RNA sequencing. Paired end sequence reads were aligned to the human genome (hg38) using Rsubread $^{55}$, (>90\% of $\sim 25 \times 10^{6}$ unique reads/sample mapped) and translated to expression counts via featurecounts, followed by a standard edgeR pipeline ${ }^{56}$ to determine differentially expressed genes (DEGs), visualized with volcano plots (ggplot2) and interpreted by gene set enrichment ${ }^{57}$ and in particular the enrichment in the Hallmarks, Curated, GO and Reactome sets was analyzed.

Similarly, in the TCGA PRAD cohort tumors in the upper and lower quartile by PPARGC1A expression were identified and DEGs established. These DEGs were combined with publicly available PGC1a ChIP-Seq data ${ }^{58}$, and the enriched regions overlapped with PGC1 $\alpha$-dependent DEGs as indicated.

Consent for publication. All authors have read and approved the contents of the manuscript and consent to its publication.

\section{Results}

Transcription factor and coregulator groups are significantly down-regulated in localized prostate cancer cohorts. We generated unique gene lists of TFs $(n=2662)$; coactivators, $($ COA; $n=766)$; corepressors, (COR; $n=599)$; and mixed function coregulators, (MIXED; $n=511$ ) (Supplementary Table S1). These groups were used to test the family-wide significance of changes in expression (mRNA or protein), CNA and mutation of each group in three local cancer (MSKCC, PRAD, OICR) and four advanced (MICH, FH, NEURO and SU2C) cancer cohorts (Table 1).

Hypergeometric tests revealed that COAS, CORS and MIXED genes, but not TFs, were significantly protected $(\mathrm{p}<0.01)$ from loss of function structural variation in normal tissues $\left(\mathrm{GNOMAD}^{59}\right)$ and significantly enriched in Pan-Cancer fitness genes ${ }^{60}$.

Next, a bootstrapping permutation approach was used to test if observed family-wide changes were more than predicted by chance ${ }^{61}$. We calculated empirical p-values based on the position of the family gene set (e.g. TF, COA etc.) relative to the distribution of random gene sets of the same size. The heatmap in Supplementary Figure S1A shows CORS expression in the TCGA cohort, indicating the genes are commonly down-regulated. To test CORS down-regulation we identified the proportion of these genes altered by $>2 \mathrm{Z}$-scores (vertical dashed red line, Supplementary Figure S1A, right panel) and compared this to the proportion of all gene families downregulated in sets of the same size (green histogram); a similar analyses was applied to the up-regulated genes (red histogram). The observed proportion of CORS down-regulated by $>2$ Z-scores was significantly more (and hence, to the right) than the estimated proportion of down-regulated groups of the same size and randomly sampled $\left(\mathrm{p}=1 \mathrm{e}^{-05}\right)$. The CNA data and normalized exome mutation rate were treated in the same manner. Positive controls included gene groups known to be significantly altered by expression (nuclear hormone receptor (NR) down-regulation; HOX family (HOX) ${ }^{61}$ up-regulation), mutation (Cosmic_mutant) (Supplementary Figure S1B) or CNA (COSMIC_CNA) (data not shown) ${ }^{62}$.

To visualize significant results, we plotted the negative $\log _{10}$ of the FDR-corrected empirical pvalues for each test across cohorts (Fig. 1). The mRNA expression in MSKCC and TCGA, and protein expression in OICR were significantly more down-regulated and/or less up-regulated. For example, CORS were significantly more down-regulated (MSKCC, TCGA, FH) and less up-regulated (MSKCC, FH, OICR) than predicted by chance. Indeed, all groups were more down-regulated or less-upregulated in at least 4 cohorts. Only two group/cohort tests revealed significant up-regulation; COR genes in MICH, and MIXED genes in OICR.

Therefore down-regulation of these groups was more common than predicted by chance in local tumors. The OICR cohort displayed significant protein down-regulation of TFs, although the protein detection of all genes is significantly reduced in number (Table 1 ) and therefore caution is needed to compare between patterns of 


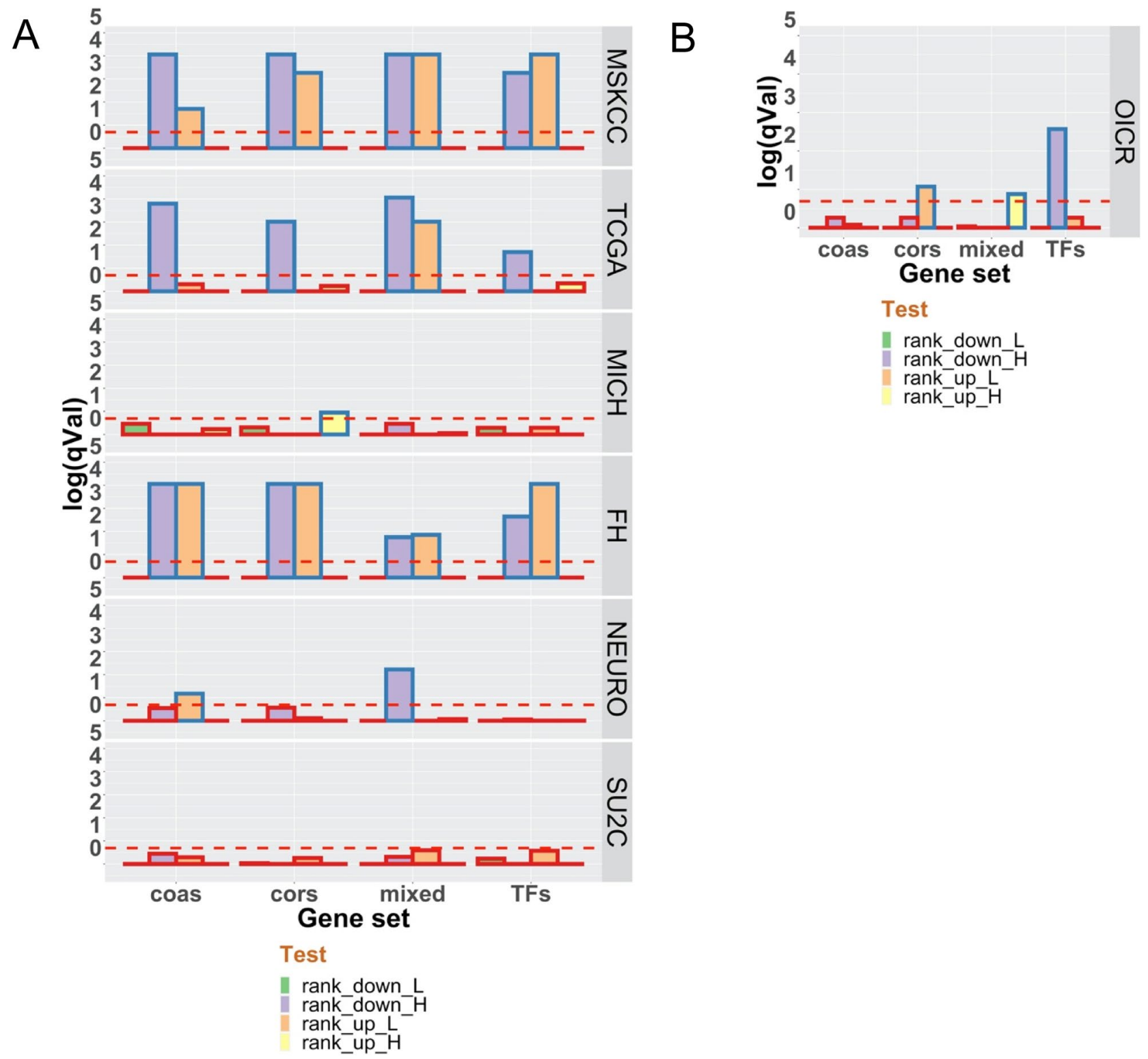

Figure 1. Family-wide analyses of transcription factors (TFs), coactivators (COAS), corepressors (CORS) and mixed function coregulators (MIXED) in PCa cohorts. The mean rank of the genes in each family were compared to the distribution of ranking of the same number of genes randomly sampled 100,000 times. The empirical p-values were - $\log 10$ transformed and FDR corrected for mRNA expression (A) and proteins (B).

RNA and protein expression. Nonetheless, the correlation between RNA (TCGA or SU2C) and protein (OICR) when considering RNA transcripts for detected proteins ranged from r 0.4 to 0.55 (Supplementary Figure S2).

By contrast to the findings on RNA and protein expression, the mutation and CNA data were largely negative; COAS and MIXED genes were more mutated than predicted by chance in the FH cohort only (Supplementary Figure S1C). These findings suggest that changing the stoichiometry of TF and coregulator interactions is potentially more impactful in local tumors than disruption through either mutation or CNA.

Reduced expression of transcription factors and coregulators associates with more aggressive PCa. Next, from each of the 15 significant RNA expression-cohort relationships we identified those genes with the most frequent and greatest expression change. For example, COAS in the TCGA cohort, were filtered for genes altered by $>2$ Z-scores in 35\% of TCGA tumors. The filtered RNA (Fig. 2) and protein expression (Supplementary Figure S3) were visualized as heatmaps.

Shared targets between the TCGA and MSKCC cohorts were investigated further (Fig. 3A). PubMed analyses (search terms given in Table Legend) revealed an uneven representation of these genes in the context of $\mathrm{PCa}$ (Table 2). These genes included those known to be commonly altered, including ERG, a frequently upregulated a TF in $\mathrm{PCa}^{35}$ and common target of translocations with TMPRRS2 ${ }^{63,64}$. By contrast, to date, the 


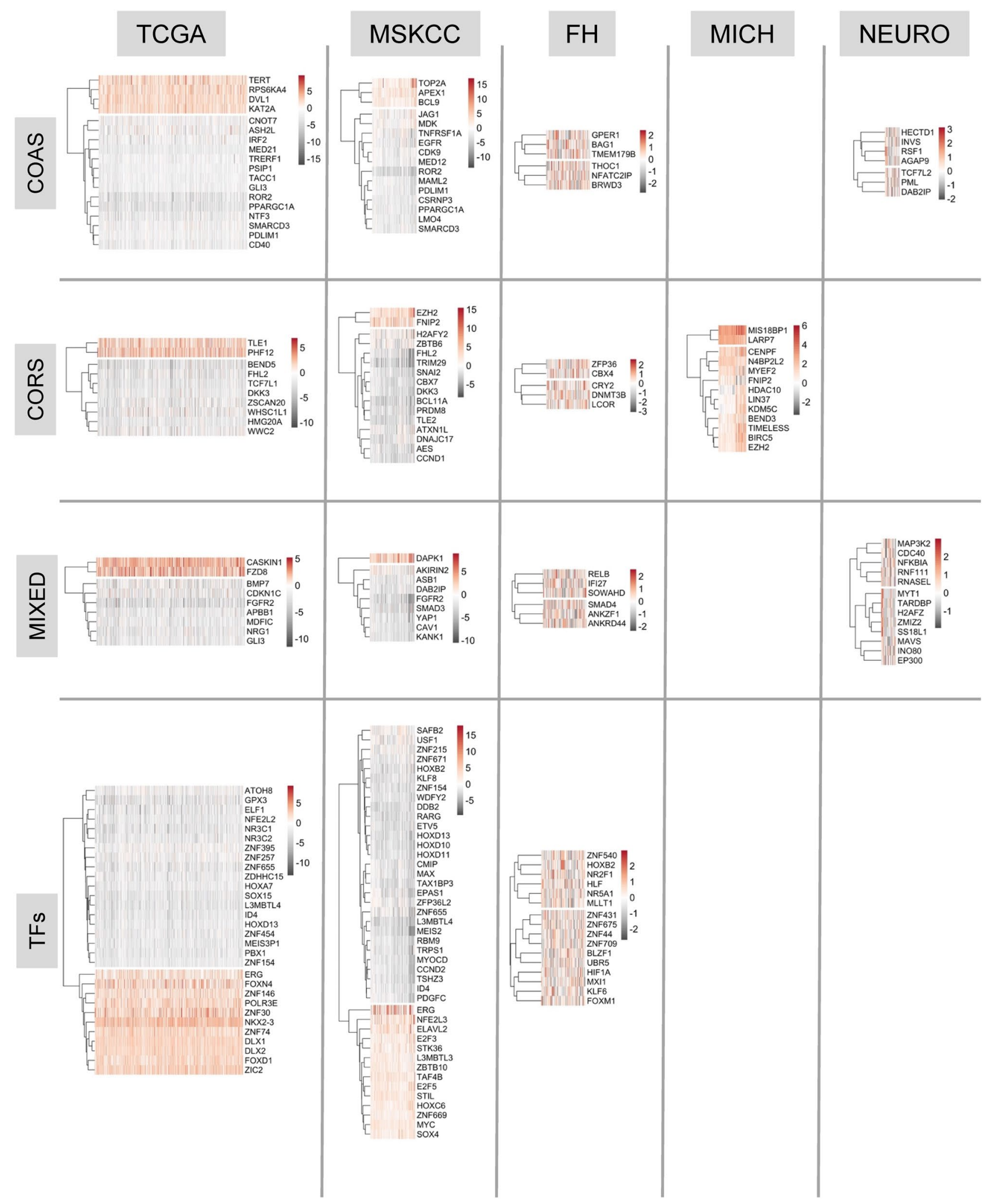

Figure 2. Expression of transcription factors (TFs), coactivators (COAS), corepressors (CORS) and mixed function coregulators (MIXED) in PCa cohorts. Genes in each group were filtered (genefilter; $>2.5 \mathrm{Z}$ scores in $35 \%$ tumors) to reveal the most frequently and strongly altered and genes and tumors (columns) were visualized (pheatmap). 

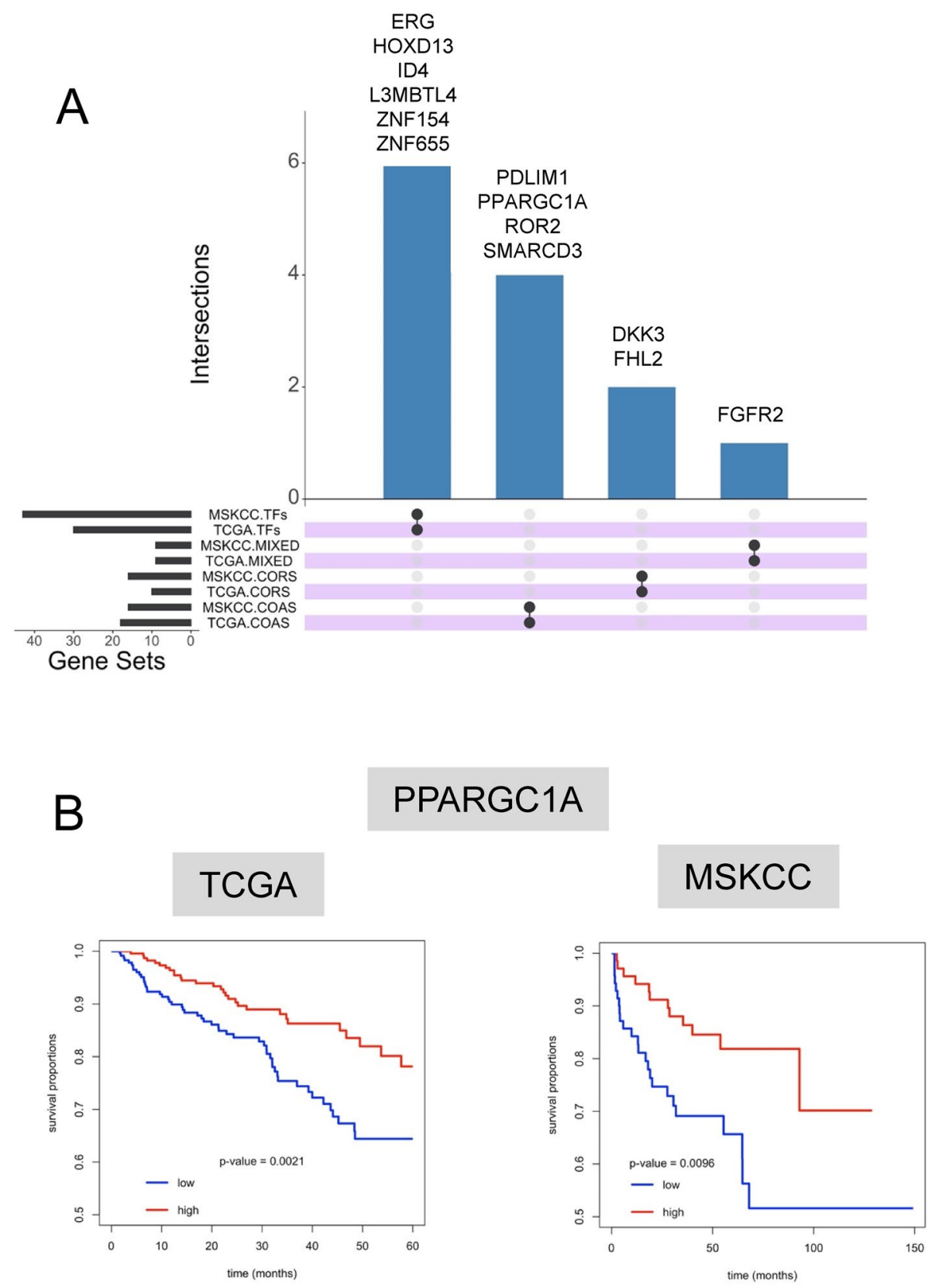

Figure 3. Identification of commonly altered transcription factors (TFs), coactivators (COAS), corepressors (CORS) and mixed function coregulators (MIXED) in the MSKCC and TCGA cohorts. (A) Genes commonly altered in both cohorts were identified and visualized (UpsetR). (B) Kaplan-Meier plots of the relationship between tumors with lower and upper quartile PPARGC1A expression and time to biochemical progression.

COA, PDLIM1 has not been investigated in the context of PCa, and others such as PPARGC1A have only been modestly investigated ${ }^{38,65-70}$.

Next, we examined how expression related to PCa progression by generating Kaplan Meier estimates of the time to biochemical progression (Table 3). In both TCGA and MSKCC cohorts, PPARGC1A was the only gene to be commonly and significantly down-regulated, and significantly associated with reduced time to biochemical progression (Fig. 3B).

Knockdown of PGC1 $\alpha$ increases proliferation and alters a large transcriptome. We generated stable PGC1 $\alpha$ knockdown clones in LNCaP cells and examined the cell phenotype, and basal and PPAR $\gamma$ ligand ETYA transcriptome $\mathrm{F}^{71,72}$. (Fig. 4A). Reduced PGC1 $\alpha$ expression increased the basal cell proliferation rate compared to vector controls, but not the anti-proliferative effect of ETYA (Fig. 4B). Reduced PGC1a also increased the invasiveness of the cells as measured by a scratch assay (Fig. 4C). 


\begin{tabular}{|l|l|l|}
\hline Shared genes & Group & Publications \\
\hline ERG & TFs & 1057 \\
\hline HOXD13 & TFs & 20 \\
\hline ID4 & TFs & 0 \\
\hline L3MBTL4 & TFs & 3 \\
\hline ZNF154 & TFs & 0 \\
\hline ZNF655 & TFs & 0 \\
\hline PDLIM1 & COA & 0 \\
\hline PPARGC1A & COA & 8 \\
\hline ROR2 & COA & 4 \\
\hline SMARCD3 & COA & 0 \\
\hline DKK3 & COR & 13 \\
\hline FHL2 & COR & 16 \\
\hline FGFR2 & MIXED & 45 \\
\hline
\end{tabular}

Table 2. Number of publications addressing the top altered genes in both the TCGA and MSKCC cohorts. The genes were mined in PubMed using the following search term (prostate cancer [Title/Abstract]) AND gene name [Title/Abstract]).

\begin{tabular}{|l|l|l|l|}
\hline Gene & Group & TCGA.Adj.Pval & MSKCC.Adj.Pval \\
\hline KAT2A & COAS & 0.001 & NS \\
\hline PPARGC1A & COAS & 0.057 & 0.061 \\
\hline NTF3 & COAS & 0.057 & NS \\
\hline MED21 & COAS & 0.057 & NS \\
\hline ELF1 & TFs & 0.059 & NS \\
\hline ZNF655 & TFs & NS & 0.034 \\
\hline LMO4 & COAS & NS & 0.034 \\
\hline MAML2 & COAS & NS & 0.034 \\
\hline HOXD11 & TFs & NS & 0.034 \\
\hline MYOCD & TFs & NS & 0.034 \\
\hline TSHZ3 & TFs & NS & 0.034 \\
\hline SNAI2 & CORS & NS & 0.047 \\
\hline HOXD10 & TFs & NS & 0.047 \\
\hline MEIS2 & TFs & NS & 0.049 \\
\hline FHL2 & CORS & NS & 0.061 \\
\hline FGFR2 & MIXED & NS & 0.061 \\
\hline TRIM29 & CORS & NS & 0.061 \\
\hline CAV1 & MIXED & NS & 0.061 \\
\hline CCND2 & TFs & NS & 0.061 \\
\hline ID4 & TFs & NS & 0.071 \\
\hline HOXC6 & TFs & NS & 0.071 \\
\hline SAFB2 & TFs & NS & 0.087 \\
\hline HOXD13 & TFs & NS & 0.094 \\
\hline & & & \\
\hline
\end{tabular}

Table 3. Relationships between altered expression and time to disease progression. Kaplan-Meier plots of the relationship between tumors with lower and upper quartile expression of the indicated gene and time to biochemical progression were generated and the FDR-corrected $\mathrm{p}$ values indicated.

RNA-Seq revealed the impact of reduced PGC1 1 expression in LNCaP cells had a profound impact on gene expression, reflecting the significant impact on proliferation the knockdown of PGC1a (Fig. 4D,E). These changes included 37 microRNA, 243 intra.microRNA, 343 lncRNA and 3600 protein coding genes significantly altered. By contrast the impact of ETYA was very modest. This suggests that PGC1a regulation of ligand-activated PPAR $\gamma$ does not appear to be significant, at least with respect to the impact of ETYA exposure (Fig. 4D).

Supporting a coactivator function, PGC1a knockdown down-regulated more than up-regulated genes. For example, 30 miRNA were down-regulated (e.g. miR17 host gene) and only 7 up-regulated, and similarly 1993 protein-coding genes were down-regulated and 1607 up-regulated. The fold change was also skewed to downregulation, with the mean $\operatorname{logFC}$ for down-regulated protein-coding genes being -1.11 , and 0.83 for up-regulated genes and similarly the mean $\log F C$ for down-regulated lncRNA was -1.20 , and 0.87 for up-regulated genes 

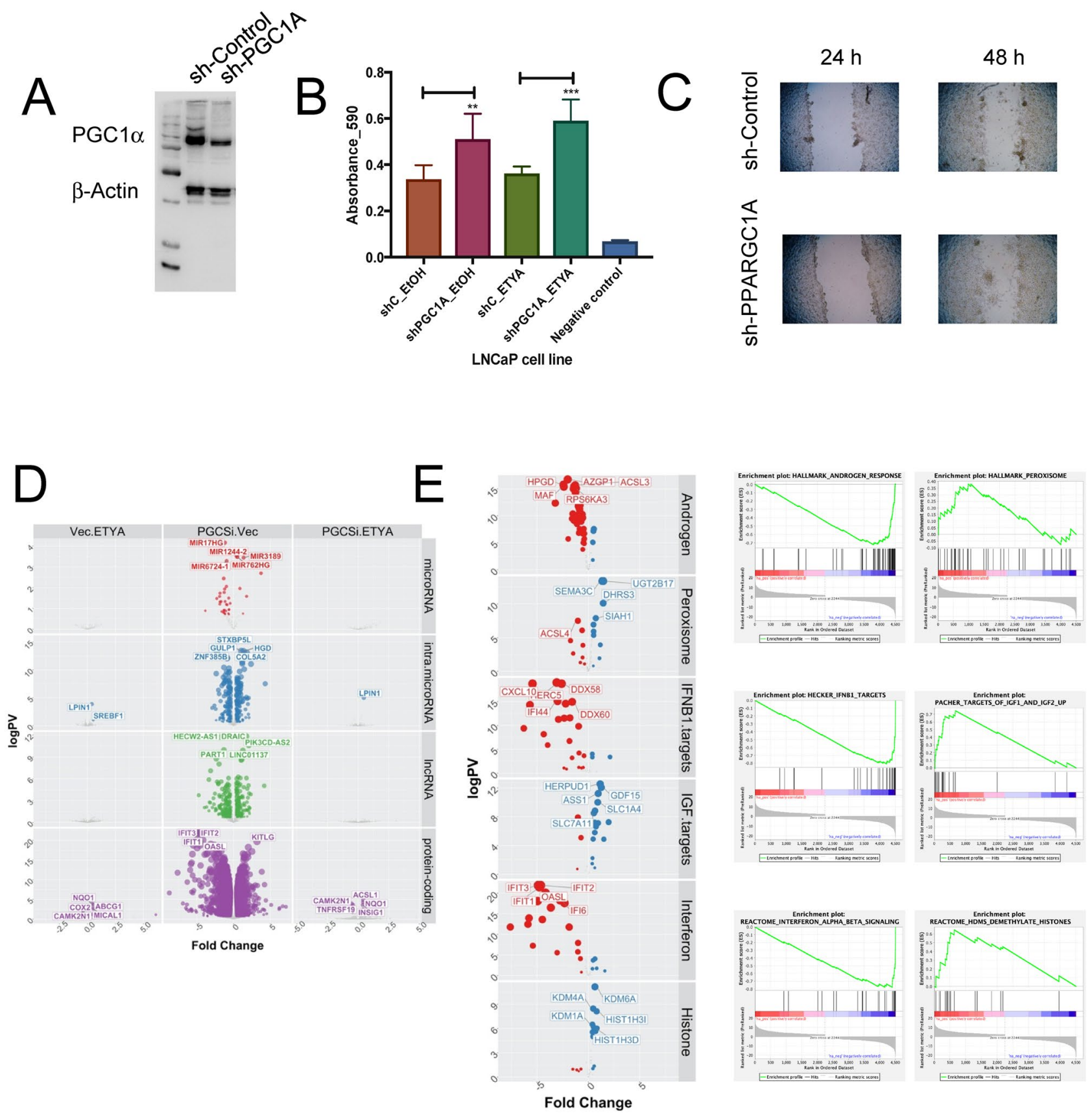

Figure 4. Stable knockdown of PGC1a in LNCaP cells changes proliferation and gene expression patterns. (A) LNCaP cells were each stably transfected with two shRNA constructs (sh-PGC1A 34 and 35) targeting PPARGC1A resulting in reduced PGC1 $\alpha$ expression (sh-PGC1A) compared to empty vector (sh-CTRL) at protein level as detected in western blotting. (B) Measurements of cellular levels of ATP, as an indicator of cell viability was detected in the vector controls and knockdown cells. Each measurement was performed in biological triplicates and in triplicate wells. Cells were treated in triplicate with exogenous PPAR $\gamma$ ligand ETYA $(10 \mu \mathrm{M}, 96 \mathrm{hr})$ or EtOH vehicle control. Increased cell proliferation was seen in sh_PGC1A cells after treatment with ETYA at 96 h. (C) Time course scratch closure of sh-Control and sh-PPARGC1A cells mechanically wounded with p200 sterile pipette tip, sh-PPARGC1A after $48 \mathrm{~h}$ showed increased cell migration compared to sh-Control. (D) LNCaP sh-PGC1A and sh-CTRL cells were treated with ETYA $(10 \mu \mathrm{M}, 24 \mathrm{~h})$ or EtOH vehicle control and total RNA expression and RNA-Seq undertaken according to the edgeR pipeline. Volcano plots depicting expression changes upon PGCl $\alpha$ knockdown or in response ETYA in the indicated classes of RNA in color $(-\log 10$ (p.adj) $>1$, abs (log2(fold change) )). (E) Summary of significantly enriched pathways from gene set enrichment analyses (GSEA) (FDR q.val<0.05) associated with reducing PGC1 $\alpha$ expression levels. 


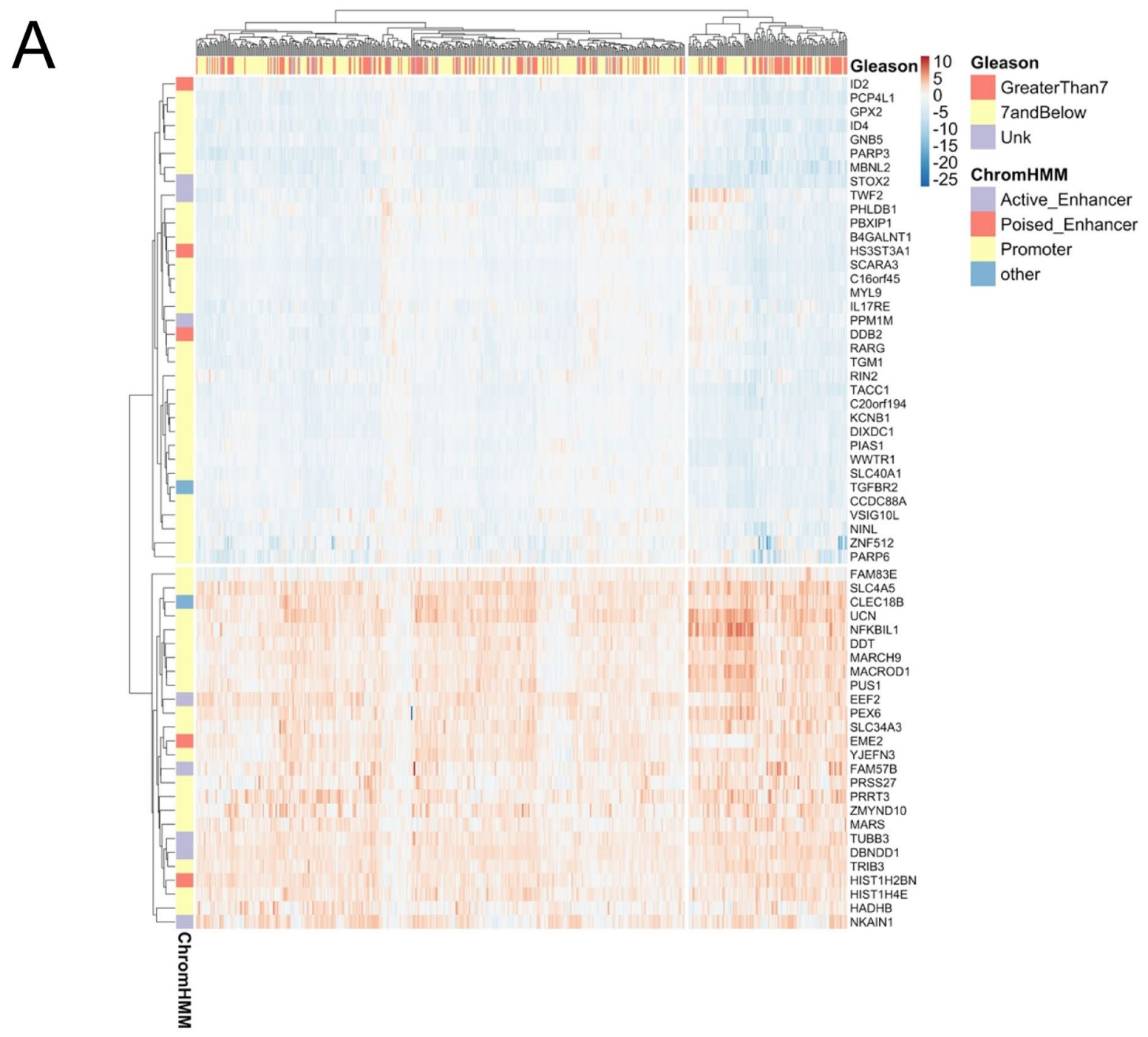

B

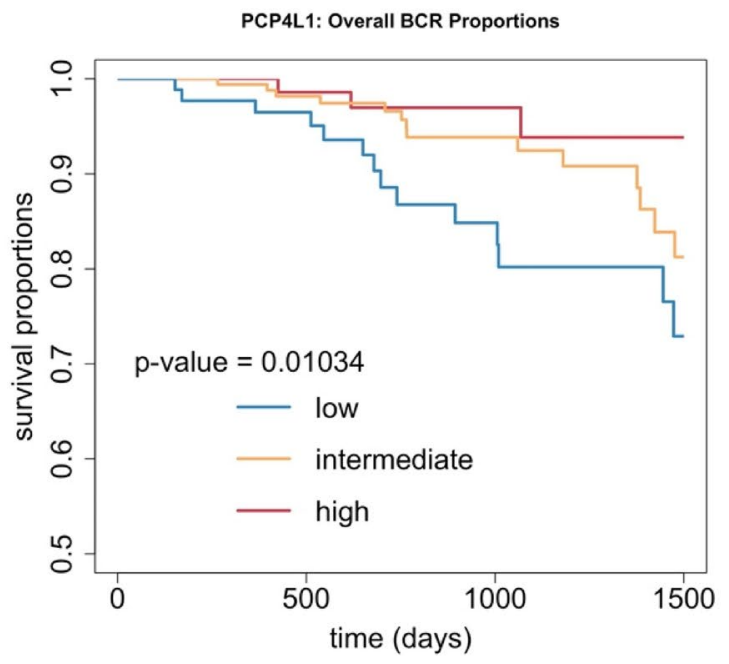

FAM57B: Overall BCR Proportions

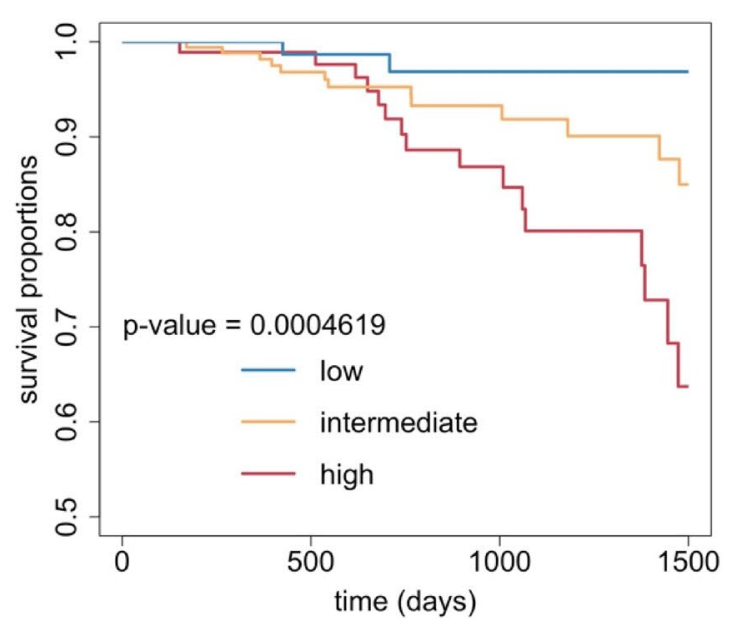


४ Figure 5. Expression of PGC1 $\alpha$-regulated and PGC1 $\alpha$-bound genes in the TCGA cohort. (A) PGC1 $\alpha$ ChIPSeq data (GSE75193) were annotated to genes within $100 \mathrm{~kb}$ to yield 2381 PGC1 a peak:gene relationships that were filtered to include only those that overlapped by 1 bp with chromatin states in LNCaP derived using ChromHMM to yield PGC1 a peak:gene relationships. In turn these further filtered to include only those genes that were; 1. differentially regulated by PPARGC1A shRNA in LNCaP cells ( $\mathrm{n}=4187$, Fig. 4$) ; 2$. differentially expressed in the TCGA cohort between the top and bottom quartile expressing PPARGC1A expression $(\mathrm{n}=7324)$, resulting in 160 genes that were significantly bound by PGC1 $\alpha$ and whose expression was significantly altered by both shRNA PPARGC1A in LNCaP cells and significantly altered in TCGA tumors in PPARGC1A-dependent manner. The top 63 genes are illustrated (pheatmap) and their expression patterns clustered higher Gleason grade tumors (X-squared $=5.0601$, p-value $=0.025)$. (B) Kaplan-Meier plots of the relationship between tumors with lower and upper quartile expression of the indicated genes and time to biochemical progression.

(Fig. 4D). LISA cistrome analyses ${ }^{73}$ of the PGC1 1 -dependent genes (Supplementary Figure S4) revealed that the top 50 transcription factors associated with these DEGs included 5 nuclear receptors (AR, NR3C1, ESR1, PR, PPARG) as well as known pioneer and lineage-determining transcription factors implicated in PCa, including FOXA1, GATA2, HOXB13, FOXA2, CEBPB and SOX4.

We undertook GSEA analyses to classify the PGC1 $\alpha$-dependent changes in gene expression ${ }^{74}$. Supplementary Figure S5 illustrates the top eight positive and negative significant $($ FDR $<0.1)$ normalized enrichment scores (NES) in four gene set categories; Hallmarks, Curated, GO and Reactome sets. These highly enriched gene patterns illuminate the cancer biology impact of reduced PGC1a expression. The NES plots and expression of the altered genes in each term is shown in Fig. 4E.

Analyses of the Hallmark gene sets revealed androgen response genes were negatively and peroxisome genes positively enriched, respectively, suggesting that PGC1a expression represses AR signaling and enhances the activity of the peroxisome. AR target genes notably associated with energy production were repressed, including the putative tumor suppressor hydroxyprostaglandin dehydrogenase $(H P G D)^{75}$, as well as Acyl-CoA synthetase long chain family member 3 (ACSL3) $)^{76}$ and Alpha-2-glycoprotein 1, zinc-binding $(A Z G P 1)^{77}$. Up-regulated peroxisome genes included retinoic acid anabolizing enzyme, Dehydrogenase/Reductase $3(D H R S 3)^{78}$, and the steroid catabolizing enzyme UDP Glucuronosyltransferase Family 2 Member B17 (UGT2B17) ${ }^{79}$. These changes in hallmark gene sets suggests that altered PGC1 $\alpha$ disrupts the energetic utilization of the cell and distorts AR signaling.

This was also borne out by changes in Curated gene sets, with repression of Interferon signaling genes including retinoic acid inducible DExD/H-Box Helicase $58(D D X 58)^{80}$, and upregulation of IGF-signaling gene targets including the AR-target genes Homocysteine Inducible ER Protein With Ubiquitin Like Domain 1 (HERPUD1) ${ }^{81}$ and Growth Differentiation Factor 15 (GDF15), known to be a prostate mitogen ${ }^{82}$. Similarly, Reactome gene set enrichment revealed repression of Interferon regulated genes including Interferon Induced Protein With Tetratricopeptide Repeats 1 (IFIT1 $)^{83}$, which is actually repressed by PPARa ${ }^{84}$. Interestingly, this was accompanied by upregulation of a number of histone modifying enzymes including lysine demethylases ${ }^{84}(K D M 1 A, K D M 4 A$, KDM6A) suggesting a potential for significant changes in the methylation status of histone lysine modifications such as $\mathrm{H} 3 \mathrm{~K} 4 \mathrm{me} 3$ and $\mathrm{H} 3 \mathrm{~K} 27 \mathrm{me} 3$, and thereby impacting the epigenome.

Genes bound and regulated by PGC1 $\alpha$ associate with more aggressive PCa. Reduced PGC1 $\alpha$ expression impacted gene expression positively and negatively. Whether PGC1 $\alpha$ exerts both coactivator and corepressor functions are obscured by direct (cis) and indirect (trans) relationships between PGC1 $\alpha$ and the regulated genes captured by RNA-Seq. To identify PGC1a cis-dependent gene expression we examined how genes bound by PGC1 $\alpha$ binding relate to changes in gene expression.

PGC1a ChIP-Seq data ${ }^{58}$ were combined with the PGCla-dependent gene expression patterns and shared genes were identified. Given each protein-coding genes is regulated by multiple enhancers ${ }^{85,86}$ and promotercapture $\mathrm{Hi}-\mathrm{C}$ experiments revealed the median distance between enhancer and target gene is $158 \mathrm{~kb}^{87}$. Therefore PGC1a ChIP-Seq peaks were segregated into different chromatin states identified in LNCaP cells (e.g. Promoter, Active Enhancer) ${ }^{88}$, and annotated to known genes within $100 \mathrm{~kb}$. Thus, the 1304 PGC1a ChIP-Seq peaks overlapped with 914 chromatin states identified in LNCaP, and annotated to 2381 peak:gene relationships.

In the first instance we examined how PPARGC1A correlated either to genes that were differentially regulated by PGC1 $\alpha$ knockdown in LNCaP cells $(n=4187$, Fig. 4D) and bound by PGC1 $\alpha$ overlapping with LNCaP chromatin states $(\mathrm{n}=2381)$. We compared the relationships between PGC1 $\alpha$ binding and gene expression in the TCGA cohort. In the first instance cumulative correlation plots were generated, which revealed that the empirical correlation between the expression of PPARGC1A and genes regulated by PPARGC1A shRNA were significantly more positive than the background of all expressed genes (KS test; $p<1 e-9$ ) (Supplementary Figure S6, green symbol). This suggests that there is a stronger positive correlation between PGC1 $\alpha$ expression and PGC1a-dependent genes than predicted by chance, and fits with a model of coactivator function. By contrast, the correlation between the expression of PPARGC1A and genes directly bound by PGC1 1 were significantly more negative than predicted by chance and suggested the correlations were significantly more negative (KS test; $\mathrm{p}<1 \mathrm{e}-9)$. This suggests that direct binding of PGC1 $\alpha$ is more nuanced in its direct relationship with gene levels, with a more even distribution of negative and positive correlations with expression.

Next, these 2381 PGC1 $\alpha$ peak:gene relationships linked to LNCaP chromatin states were filtered to include genes that were: (1) differentially regulated by PPARGC1A shRNA in LNCaP cells ( $\mathrm{n}=4187$, Fig. 4); (2) differentially expressed in the TCGA cohort between the top and bottom quartile expressing PPARGC1A expression $(\mathrm{n}=7324)$. This identified 160 genes bound by PGC1 $\alpha$ and significantly altered by PGC1 $\alpha$ knockdown in LNCaP 
cells and significantly altered PPARGC1A-dependent expression in TCGA, with the most altered 63 genes in the TCGA cohort shown in Fig. 5A. Expression genes clustered higher Gleason grade tumors (X-squared $=5.0601$, p-value $=0.025$ ). Interestingly, amongst the PGC1 $\alpha$-dependent gene signature 25 genes were upregulated, and 38 genes were actually downregulated, again suggesting that the direct impact of PGC1 $\alpha$ on gene expression is more nuanced and not exclusively a coactivator. Finally, we screened how expression of these 63 PGC1a-dependent genes related to tumor outcome by generating Kaplan-Meier plots. The top five significant genes were FAM57B, EME2, DDB2, PCP4L1 and PBXIP1 and the plots for an upregulated gene (FAM57B) and a downregulated gene (PCP4L1) are shown in Fig. 5B. Interestingly, FAM57B is an established PGC1 $\alpha$ target gene that regulates adipocyte differentiation ${ }^{89}$. Similarly, $P C P 4 L 1$ is related to obesity induced phenotypes ${ }^{90}$ and $P B X I P 1$ plays a role in regulation of differentiation ${ }^{91}$ in part by regulating ERa signaling ${ }^{92}$. By contrast EME2 and DDB2 relate to DNA repair ${ }^{93,94}$.

\section{Discussion}

The current study was undertaken to identify the most significantly altered and clinically-relevant TF and coregulators in PCa. It was reasoned that this knowledge may help to explain disease progression risks, or to highlight novel therapeutic opportunities. Assessment of family-wide significance across seven PCa cohorts revealed that TFs, COAS, CORs and MIXED were more down-regulated and less up-regulated at the mRNA and protein level, most clearly in local tumor cohorts. In contrast, family-wide CNA and mutation levels were not significant. Filtering those group/cohort relationships identified the most altered genes in the local tumor cohorts (MSKCC and TCGA). Overlapping identified commonly altered genes associated with PCa including $E R G^{35}$ (which was also altered at the protein level in the OICR cohort) and FGFR2 $2^{95}$, but also identified others that were relatively under-investigated such as PPARGC1 $A^{37,38,96}$ but associated with worse disease free survival, or uninvestigated in PCa, such as the metastasis suppressor PDLIM1 ${ }^{97}$. Stable knockdown of PGC1a increased proliferation and invasion of LNCaP cells, and profoundly altered the transcriptome. Finally, PGC1 $\alpha$ bound and regulated genes associated with higher grade tumors in the TCGA cohort and individual genes such as the PPAR $\gamma$ target gene FAM57B associated with worse disease-free survival.

The global down-regulation of TFs and co-regulators suggests that an initial event in PCa is disrupting the flexibility of gene regulatory signaling that may limit the permutations of TF co-regulator interactions or lessen the ability of the TF and co-regulators to form effective stoichiometric interactions for correct functioning. This may suggest that although these genes are reduced in expression they are not altered by structural variation, and therefore they remain functional, and in turn this may support the disrupted stoichiometry argument. The current findings would suggest that globally the flexibility of TF actions is disrupted. This may suggest that signaling is more restricted in terms of capacity, and this is seen in the PCa AR transcriptome ${ }^{27,29}$, but also in signaling via MYC and AP1 (reviewed in ${ }^{73}$ ).

The stringent filtering of these data and overlap between TCGA and MSKCC revealed commonly and significantly altered genes but are unevenly investigated in PCa (Table 2). Genes that are only identified in one cohort and whose expression is associated with more aggressive disease (Table 3) reveals a number of genes that are potentially impactful in disease but are under-investigated or have not been investigated at all in the context of PCa. PPARGC1A was selected for mechanistic study, given it was down-regulated and significantly associated with worse disease-free survival in both MSKCC and TCGA cohorts. Knockdown of PGC1a in LNCaP cells increased proliferation and migration, coupled with a profound impact on the transcriptome. The PGC1 $\alpha$ transcriptome was enriched for terms associated with AR signaling as well overlaps with regulation of the peroxisome, interferon signaling and epigenetic modifiers, as well as being significantly enriched for the regulatory actions of AR, NR3C1, PPARg and several pioneer factors. These findings were further supported when considering target genes that were bound by PGCla and differentially expressed in the TCGA cohort. These genes distinguished aggressive tumors and included RAR $\gamma / \mathrm{TACC}^{24}$, which we have previously established to antagonize AR signaling, as well as genes associated PPAR $\gamma$ signaling. Furthermore, motif analyses revealed that basic helix-loop-helix (b-HLH) motifs were enriched in the PGC1a ChromHMM sites, as were sites for LXR binding. These findings suggest that PGC1 $\alpha$ regulates signaling by a cohort of nuclear receptors including AR, RAR $\gamma, \operatorname{PAR} \gamma$ as well epigenetic process and interferon signaling. Interestingly, we examined expression of PPARGC1A in a broader panel of PCa cell lines in the Broad Cancer Cell Line Encyclopedia ${ }^{98}$, which revealed that in seven out of eight models the expression was downregulated by more than two Z-scores (Supplementary Figure S7). This is interesting given that the cell lines represent different aspects of $\mathrm{PCa}$ (either early late or stage) and specifically, which either express AR (LNCaP), or are AR negative (PC-3). PPARGC1A was also down-regulated in cells lines expressing the AR variant (AR-V7) associated with aggressive disease (22Rv1, VCaP). This suggests that down-regulation of PPARGC1A is both common and sustained in PCa cell line models.

Earlier studies by Carracedo and colleagues identified reduced expression of PGC1 $\alpha$ in PCa, and these workers pursued a strategy to over-express PGC1 $\alpha$ in advanced PCa cell (PC-3) cells line and analyzed these effects in two impactful studies ${ }^{37,38}$. Their findings support a role for PGCla to regulate a transcriptome that is regulated by PGC1 $\alpha$ and ERRa cross-talk, which regulates MYC signaling to regulate invasiveness; indeed in the current study b-HLH motifs were identified in the PGC1 $a$-binding sites identified in LNCaP cells. The PGC1a-dependent transcriptome in PC-3 cells is $\sim 900$ genes (GSE75193) and few $(n=119)$ appear to be shared with the RNA-Seq data generated in the current study, but none of the genes proposed by the authors as a PGCla-dependent signature overlap with the 63 PGC1 $\alpha$-dependent and PGC1 $\alpha$-bound genes significantly altered in TCGA cohort associated with more aggressive disease.

Thus, whilst the current study and the Carracedo studies both identify important roles for PGC1a to regulate tumor aggressiveness, the mechanisms appear to be different. This may arise for technical reasons (for example, cell background and transcriptomic approach) and may reflect the differential impact of knockdown 
versus over-expression selecting for different transcriptionaly-dependent events. Certainly, the fact that PGC1 $a-$ dependent and PGC1 $\alpha$-bound genes favor down-regulated over up-regulated genes ( 2:1) suggests that PGC1 $\alpha$ participates in diverse transcriptional events. Outside of $\mathrm{PCa}$, in renal disease it has been proposed that PGC1a determines phenotypic consequences by selecting which TFs to interact with, and that RARs/RXRs, PPARs is associated with fatty acid metabolism ${ }^{99}$. It is tempting to speculate the phenotype is current study is associated with similar energetic changes.

\section{Conclusion}

The approach applied in the current study identified both well-established factors, such as ERG, and relatively under-investigated factors, notably PPARGC1A, that associate with PCa progression risks. Indeed, the approaches and data integration are relatively generic and considers all genes in a class or superfamily to identify which may have merit to investigate. This is an important step for many wet-lab approaches, given that it can often take considerable resources to dissect a gene function. For example, the 20,000 ft view approach to cancer genomics in PCa integrates genome-wide data to identify novel networks ${ }^{18,58}$. Traditionally trained wet-lab based investigators often face challenges in assimilating these findings and may rather search for gene(s) in the supplementary data $\mathrm{S} 1$ that are from the family on which they study; this can be considered as a $200 \mathrm{ft}$ view. The current approach is neither the $200 \mathrm{ft}$ nor $20,000 \mathrm{ft}$ views, but rather a $2000 \mathrm{ft}$ view. That is, the approach allows an investigator to remain focused in their research arena, for example TF co-regulators, but ask the global question over how these are altered and at what stage of cancer progression. This is a relatively generic approach and may find traction with investigators across disease types.

\section{Data availability}

The PPARGC1A shRNA dependent RNA-Seq will be deposited on GEO.

Received: 17 August 2020; Accepted: 5 November 2020

Published online: 23 November 2020

\section{References}

1. Kerr, J. F., Wyllie, A. H. \& Currie, A. R. Apoptosis: a basic biological phenomenon with wide-ranging implications in tissue kinetics. Br. J. Cancer 26, 239-257 (1972).

2. Henry, G.H. et al. A Cellular anatomy of the normal adult human prostate and prostatic urethra. Cell Rep. 25, 3530-3542 e3535 (2018).

3. Liu, S. et al. A comprehensive analysis of coregulator recruitment, androgen receptor function and gene expression in prostate cancer. Elife 6 (2017).

4. Paltoglou, S. et al. Novel androgen receptor coregulator GRHL2 exerts both oncogenic and antimetastatic functions in prostate cancer. Cancer Res. 77, 3417-3430 (2017).

5. Wang, T. et al. Flightless I homolog represses prostate cancer progression through targeting androgen receptor signaling. Clin. Cancer Res. 22, 1531-1544 (2016).

6. Gu, H. et al. Upregulated LMO1 in prostate cancer acts as a novel coactivator of the androgen receptor. Int. J. Oncol. 47, 2181-2187 (2015).

7. Blessing, A. M. et al. Identification of a novel coregulator, SH3YL1, that interacts with the androgen receptor N-terminus. Mol. Endocrinol. 29, 1426-1439 (2015).

8. Toropainen, S. et al. SUMO ligase PIAS1 functions as a target gene selective androgen receptor coregulator on prostate cancer cell chromatin. Nucleic Acids Res. 43, 848-861 (2015).

9. Niu, Y. et al. Tissue prostate-specific antigen facilitates refractory prostate tumor progression via enhancing ARA70-regulated androgen receptor transactivation. Cancer Res. 68, 7110-7119 (2008).

10. Poukka, H., Aarnisalo, P., Santti, H., Janne, O. A. \& Palvimo, J. J. Coregulator small nuclear RING finger protein (SNURF) enhances Sp1- and steroid receptor-mediated transcription by different mechanisms. J. Biol. Chem. 275, 571-579 (2000).

11. Khanim, F. L. et al. Altered SMRT levels disrupt vitamin D3 receptor signalling in prostate cancer cells. Oncogene 23, 6712-6725 (2004).

12. Henzler, C. et al. Truncation and constitutive activation of the androgen receptor by diverse genomic rearrangements in prostate cancer. Nat. Commun. 7, 13668 (2016).

13. Chen, Z. et al. Diverse AR-V7 cistromes in castration-resistant prostate cancer are governed by HoxB13. Proc. Natl. Acad. Sci. USA 115, 6810-6815 (2018).

14. Geybels, M. S. et al. Epigenetic signature of Gleason score and prostate cancer recurrence after radical prostatectomy. Clin. Epigenet. 8, 97 (2016).

15. Nickerson, M. L. et al. TET2 binds the androgen receptor and loss is associated with prostate cancer. Oncogene 36, 2172-2183 (2017).

16. Wang, Y. et al. Roles of distal and genic methylation in the development of prostate tumorigenesis revealed by genome-wide DNA methylation analysis. Sci. Rep. 6, 22051 (2016).

17. Kang, Z., Janne, O. A. \& Palvimo, J. J. Coregulator recruitment and histone modifications in transcriptional regulation by the androgen receptor. Mol. Endocrinol. 18, 2633-2648 (2004).

18. Armenia, J. et al. The long tail of oncogenic drivers in prostate cancer. Nat. Genet. 50, 645-651 (2018).

19. Takeda, D.Y. et al. A somatically acquired enhancer of the androgen receptor is a noncoding driver in advanced prostate cancer. Cell 174, 422-432 e413 (2018).

20. Barfeld, S. J. et al. c-Myc antagonises the transcriptional activity of the androgen receptor in prostate cancer affecting key gene networks. EBioMedicine 18, 83-93 (2017).

21. Toropainen, S. et al. Global analysis of transcription in castration-resistant prostate cancer cells uncovers active enhancers and direct androgen receptor targets. Sci. Rep. 6, 33510 (2016).

22. Wang, Q. et al. Androgen receptor regulates a distinct transcription program in androgen-independent prostate cancer. Cell 138, 245-256 (2009).

23. Pomerantz, M. M. et al. The androgen receptor cistrome is extensively reprogrammed in human prostate tumorigenesis. Nat. Genet. 47, 1346-1351 (2015).

24. Long, M. D. et al. The miR-96 and RARgamma signaling axis governs androgen signaling and prostate cancer progression. Oncogene 38, 421-444 (2019). 
25. Beltran, H. et al. The role of lineage plasticity in prostate cancer therapy resistance. Clin. Cancer Res. (2019).

26. Sheahan, A. V. \& Ellis, L. Epigenetic reprogramming: a key mechanism driving therapeutic resistance. Urol. Oncol. 36, 375-379 (2018).

27. $\mathrm{Ku}, \mathrm{S}$. Y. et al. $\mathrm{Rb} 1$ and Trp53 cooperate to suppress prostate cancer lineage plasticity, metastasis, and antiandrogen resistance. Science 355, 78-83 (2017).

28. Flavahan, W.A. et al. Altered chromosomal topology drives oncogenic programs in SDH-deficient GISTs. Nature (2019).

29. Bell, C. C. et al. Targeting enhancer switching overcomes non-genetic drug resistance in acute myeloid leukaemia. Nat. Commun. 10, 2723 (2019).

30. Labbe, D. P. et al. TOP2A and EZH2 provide early detection of an aggressive prostate cancer subgroup. Clin. Cancer Res. 23, 7072-7083 (2017).

31. Rotinen, M. et al. ONECUT2 is a targetable master regulator of lethal prostate cancer that suppresses the androgen axis. Nat. Med. 24, 1887-1898 (2018).

32. Nerlakanti, N. et al. Targeting the BRD4-HOXB13 coregulated transcriptional networks with bromodomain-kinase inhibitors to suppress metastatic castration-resistant prostate cancer. Mol. Cancer Ther. 17, 2796-2810 (2018).

33. Stelloo, S. et al. Integrative epigenetic taxonomy of primary prostate cancer. Nat. Commun. 9, 4900 (2018).

34. Patten, D. K. et al. Enhancer mapping uncovers phenotypic heterogeneity and evolution in patients with luminal breast cancer. Nat. Med. 24, 1469-1480 (2018).

35. Petrovics, G. et al. Frequent overexpression of ETS-related gene-1 (ERG1) in prostate cancer transcriptome. Oncogene 24, 38473852 (2005).

36. Krishnan, A. V. et al. A glucocorticoid-responsive mutant androgen receptor exhibits unique ligand specificity: therapeutic implications for androgen-independent prostate cancer. Endocrinology 143, 1889-1900 (2002).

37. Valcarcel-Jimenez, L. et al. PGC1alpha suppresses prostate cancer cell invasion through ERRalpha transcriptional control. Cancer Res 79, 6153-6165 (2019).

38. Torrano, V. et al. The metabolic co-regulator PGClalpha suppresses prostate cancer metastasis. Nat. Cell Biol. 18, 645-656 (2016).

39. Gao, J. et al. Integrative analysis of complex cancer genomics and clinical profiles using the cBioPortal. Sci Signal 6, pl1 (2013).

40. Cerami, E. et al. The cBio cancer genomics portal: an open platform for exploring multidimensional cancer genomics data. Cancer Discov. 2, 401-404 (2012).

41. Sinha, A. et al. The proteogenomic landscape of curable prostate cancer. Cancer Cell 35, 414-427 e416 (2019).

42. Cancer Genome Atlas Research, N. The molecular taxonomy of primary prostate cancer. Cell 163, 1011-1025 (2015).

43. Taylor, B. S. et al. Integrative genomic profiling of human prostate cancer. Cancer Cell 18, 11-22 (2010).

44. Kumar, A. et al. Substantial interindividual and limited intraindividual genomic diversity among tumors from men with metastatic prostate cancer. Nat. Med. 22, 369-378 (2016).

45. Beltran, H. et al. Divergent clonal evolution of castration-resistant neuroendocrine prostate cancer. Nat. Med. 22, 298-305 (2016).

46. Robinson, D. et al. Integrative clinical genomics of advanced prostate cancer. Cell 161, 1215-1228 (2015).

47. Abida, W. et al. Genomic correlates of clinical outcome in advanced prostate cancer. Proc. Natl. Acad. Sci. USA 116, 11428-11436 (2019).

48. Leman, E. S., DeMiguel, F., Gao, A. C. \& Getzenberg, R. H. Regulation of androgen and vitamin d receptors by 1,25-dihydroxyvitamin D3 in human prostate epithelial and stromal cells. J. Urol. 170, 235-240 (2003).

49. Mi, H., Muruganujan, A., Ebert, D., Huang, X. \& Thomas, P. D. PANTHER version 14: more genomes, a new PANTHER GO-slim and improvements in enrichment analysis tools. Nucleic Acids Res 47, D419-D426 (2019).

50. Durinck, S., Spellman, P. T., Birney, E. \& Huber, W. Mapping identifiers for the integration of genomic datasets with the R/Bioconductor package biomaRt. Nat. Protoc. 4, 1184-1191 (2009).

51. Mermel, C.H. et al. GISTIC2.0 facilitates sensitive and confident localization of the targets of focal somatic copy-number alteration in human cancers. Genome Biol. 12, R41 (2011).

52. Franke, J. \& Neumann, M. H. Bootstrapping neural networks. Neural. Comput. 12, 1929-1949 (2000).

53. Conway, J. R., Lex, A. \& Gehlenborg, N. UpSetR: an R package for the visualization of intersecting sets and their properties. Bioinformatics 33, 2938-2940 (2017).

54. Kivela, T. \& Grambsch, P. M. Evaluation of sampling strategies for modeling survival of uveal malignant melanoma. Invest. Ophthalmol. Vis. Sci. 44, 3288-3293 (2003).

55. Liao, Y., Smyth, G. K. \& Shi, W. The R package Rsubread is easier, faster, cheaper and better for alignment and quantification of RNA sequencing reads. Nucleic Acids Res 47, e47 (2019).

56. Robinson, M. D., McCarthy, D. J. \& Smyth, G. K. edgeR: a Bioconductor package for differential expression analysis of digital gene expression data. Bioinformatics 26, 139-140 (2010).

57. Subramanian, A., Kuehn, H., Gould, J., Tamayo, P. \& Mesirov, J. P. GSEA-P: a desktop application for gene set enrichment analysis. Bioinformatics 23, 3251-3253 (2007).

58. Charos, A. E. et al. A highly integrated and complex PPARGC1A transcription factor binding network in HepG2 cells. Genome Res. 22, 1668-1679 (2012).

59. Scheps, K. G., Hasenahuer, M. A., Parisi, G., Targovnik, H. M. \& Fornasari, M. S. Curating the gnomAD database: report of novel variants in the globin-coding genes and bioinformatics analysis. Hum. Mutat. 41, 81-102 (2020).

60. Behan, F. M. et al. Prioritization of cancer therapeutic targets using CRISPR-Cas9 screens. Nature 568, 511-516 (2019).

61. Long, M.D. \& Campbell, M.J. Pan-cancer analyses of the nuclear receptor superfamily. Nucl. Receptor Res. 2 (2015)

62. Tate, J. G. et al. COSMIC: the catalogue of somatic mutations in cancer. Nucleic Acids Res. 47, D941-D947 (2019).

63. Sanda, M.G. et al. Association between combined TMPRSS2:ERG and PCA3 RNA urinary testing and detection of aggressive prostate cancer. JAMA Oncol. (2017).

64. Tomlins, S. A. et al. Recurrent fusion of TMPRSS2 and ETS transcription factor genes in prostate cancer. Science 310, 644-648 (2005).

65. Tennakoon, J. B. et al. Androgens regulate prostate cancer cell growth via an AMPK-PGC-1alpha-mediated metabolic switch. Oncogene 33, 5251-5261 (2014).

66. Yu, S. et al. Orphan nuclear receptor estrogen-related receptor-beta suppresses in vitro and in vivo growth of prostate cancer cells via p21(WAF1/CIP1) induction and as a potential therapeutic target in prostate cancer. Oncogene 27, 3313-3328 (2008).

67. Shiota, M. et al. Peroxisome proliferator-activated receptor gamma coactivator-1alpha interacts with the androgen receptor (AR) and promotes prostate cancer cell growth by activating the AR. Mol. Endocrinol. 24, 114-127 (2010).

68. Kresovich, J. K. et al. Promoter methylation of PGC1A and PGC1B predicts cancer incidence in a veteran cohort. Epigenomics 10, 733-743 (2018).

69. Dwyer, M. A. et al. WNT11 expression is induced by estrogen-related receptor alpha and beta-catenin and acts in an autocrine manner to increase cancer cell migration. Cancer Res. 70, 9298-9308 (2010).

70. Valcarcel-Jimenez, L. et al. PGC1alpha suppresses prostate cancer cell invasion through ERRalpha transcriptional control. Cancer Res. (2019).

71. Vu-Dac, N. et al. Fibrates increase human apolipoprotein A-II expression through activation of the peroxisome proliferatoractivated receptor. J. Clin. Invest. 96, 741-750 (1995). 
72. Battaglia, S. et al. Elevated NCOR1 disrupts PPARalpha/gamma signaling in prostate cancer and forms a targetable epigenetic lesion. Carcinogenesis 31, 1650-1660 (2010).

73. Qin, Q. et al. Lisa: inferring transcriptional regulators through integrative modeling of public chromatin accessibility and ChIP-seq data. Genome Biol. 21, 32 (2020).

74. Subramanian, A. et al. Gene set enrichment analysis: a knowledge-based approach for interpreting genome-wide expression profiles. Proc. Natl. Acad. Sci. USA 102, 15545-15550 (2005).

75. Huang, X. et al. miR-620 promotes tumor radioresistance by targeting 15-hydroxyprostaglandin dehydrogenase (HPGD). Oncotarget 6, 22439-22451 (2015).

76. Migita, T. et al. ACSL3 promotes intratumoral steroidogenesis in prostate cancer cells. Cancer Sci 108, 2011-2021 (2017).

77. Cao, R. et al. AZGP1 is androgen responsive and involved in AR-induced prostate cancer cell proliferation and metastasis. J. Cell Physiol. 234, 17444-17458 (2019).

78. Lochbaum, R. et al. Retinoic acid signalling adjusts tight junction permeability in response to air-liquid interface conditions. Cell Signal 65, 109421 (2020).

79. Zhu, Z. et al. Loss of dihydrotestosterone-inactivation activity promotes prostate cancer castration resistance detectable by functional imaging. J. Biol. Chem. 293, 17829-17837 (2018).

80. Chowdhari, S. \& Saini, N. Gene expression profiling reveals the role of RIG1 like receptor signaling in p53 dependent apoptosis induced by PUVA in keratinocytes. Cell Signal 28, 25-33 (2016).

81. Segawa, T. et al. Androgen-induced expression of endoplasmic reticulum (ER) stress response genes in prostate cancer cells. Oncogene 21, 8749-8758 (2002).

82. Wang, W. et al. Prostate cancer promotes a vicious cycle of bone metastasis progression through inducing osteocytes to secrete GDF15 that stimulates prostate cancer growth and invasion. Oncogene 38, 4540-4559 (2019).

83. Pidugu, V. K. et al. IFIT1 and IFIT3 promote oral squamous cell carcinoma metastasis and contribute to the anti-tumor effect of gefitinib via enhancing p-EGFR recycling. Oncogene 38, 3232-3247 (2019).

84. Janssen, A. W. et al. The impact of PPARalpha activation on whole genome gene expression in human precision cut liver slices. BMC Genomics 16, 760 (2015).

85. Roadmap Epigenomics, C. et al. Integrative analysis of 111 reference human epigenomes. Nature 518, 317-330 (2015).

86. Thurman, R. E. et al. The accessible chromatin landscape of the human genome. Nature 489, 75-82 (2012).

87. Jung, I. et al. A compendium of promoter-centered long-range chromatin interactions in the human genome. Nat Genet 51, 1442-1449 (2019).

88. Taberlay, P. C. et al. Three-dimensional disorganization of the cancer genome occurs coincident with long-range genetic and epigenetic alterations. Genome Res 26, 719-731 (2016).

89. Yamashita-Sugahara, Y. et al. Fam57b (family with sequence similarity 57 , member B), a novel peroxisome proliferator-activated receptor gamma target gene that regulates adipogenesis through ceramide synthesis. J. Biol. Chem. 288, 4522-4537 (2013).

90. Kluth, O. et al. Identification of four mouse diabetes candidate genes altering beta-cell proliferation. PLoS Genet 11, e1005506 (2015).

91. Manavathi, B. et al. Functional regulation of pre-B-cell leukemia homeobox interacting protein 1 (PBXIP1/HPIP) in erythroid differentiation. J. Biol. Chem. 287, 5600-5614 (2012).

92. Wang, X. et al. The estrogen receptor-interacting protein HPIP increases estrogen-responsive gene expression through activation of MAPK and AKT. Biochim. Biophys. Acta 1783, 1220-1228 (2008).

93. Matsumoto, S. et al. DNA damage detection in nucleosomes involves DNA register shifting. Nature 571, 79-84 (2019).

94. Techer, H. et al. Signaling from Mus81-Eme2-dependent DNA damage elicited by Chk1 deficiency modulates replication fork speed and origin usage. Cell Rep. 14, 1114-1127 (2016).

95. Leung, H. Y. et al. Keratinocyte growth factor expression in hormone insensitive prostate cancer. Oncogene 15, 1115-1120 (1997).

96. Canto, P. et al. PPARGC1A and ADIPOQ polymorphisms are associated with aggressive prostate cancer in Mexican-Mestizo men with overweight or obesity. Cancer Biomark 19, 297-303 (2017).

97. Huang, Z. et al. PDLIM1 inhibits tumor metastasis through activating hippo signaling in hepatocellular carcinoma. Hepatology (2019).

98. Barretina, J. et al. The Cancer Cell Line Encyclopedia enables predictive modelling of anticancer drug sensitivity. Nature 483, 603-607 (2012).

99. Li, S. Y. \& Susztak, K. The role of peroxisome proliferator-activated receptor gamma coactivator 1alpha (PGC-1alpha) in kidney disease. Semin. Nephrol. 38, 121-126 (2018).

\section{Author contributions}

Approaches and design were conceived by MJC; bioinfomatic analyses was undertaken by MJC and MDL; Results were interpreted by MJC, CLB, EW and DAL; all authors contributed to manuscript preparation.

\section{Funding}

MJC acknowledges support in part from the Prostate program of the Department of Defense Congressionally Directed Medical Research Programs [W81XWH-14-1-0608], the National Institute of Health Cancer Center Support Grant (P30CA016058) to the OSUCCC The James. EW and MJC acknowledge seed-funding support from the Molecular Carcinogenesis program of the OSUCCC The James, CCSG. CLB, MJC, DAL acknowledge support in part from Prostate Cancer, UK [RIA18-ST2-022]. EAM acknowledges support The Ohio State University Translational Data Analytics Institute, startup funds from The Ohio State University.

\section{Competing interests}

The author certifies that he has NO affiliations with or involvement in any organization or entity with any financial interest (such as honoraria; educational grants; participation in speakers' bureaus; membership, employment, consultancies, stock ownership, or other equity interest; and expert testimony or patent-licensing arrangements), or non-financial interest (such as personal or professional relationships, affiliations, knowledge or beliefs) in the subject matter or materials discussed in this manuscript.

\section{Additional information}

Supplementary information is available for this paper at https://doi.org/10.1038/s41598-020-77055-5.

Correspondence and requests for materials should be addressed to M.J.C.

Reprints and permissions information is available at www.nature.com/reprints. 
Publisher's note Springer Nature remains neutral with regard to jurisdictional claims in published maps and institutional affiliations.

(c) (i) Open Access This article is licensed under a Creative Commons Attribution 4.0 International License, which permits use, sharing, adaptation, distribution and reproduction in any medium or format, as long as you give appropriate credit to the original author(s) and the source, provide a link to the Creative Commons licence, and indicate if changes were made. The images or other third party material in this article are included in the article's Creative Commons licence, unless indicated otherwise in a credit line to the material. If material is not included in the article's Creative Commons licence and your intended use is not permitted by statutory regulation or exceeds the permitted use, you will need to obtain permission directly from the copyright holder. To view a copy of this licence, visit http://creativecommons.org/licenses/by/4.0/.

(c) The Author(s) 2020 